\title{
Natural convection flow of a fluid using Atangana and Baleanu fractional model
}

\author{
Sidra Aman ${ }^{1}$, Thabet Abdeljawad ${ }^{2,3,4^{*}}$ (1) and Qasem Al-Mdallal ${ }^{* *}$
}

\section{"Correspondence:}

tabdeljawad@psu.edu.sa:

q.almdallal@uaeu.ac.ae

'Department of Mathematical

Sciences, UAE University, Al Ain, United Arab Emirates

${ }^{2}$ Department of Mathematics and General Sciences, Prince Sultan University, Riyadh, Saudi Arabia Full list of author information is available at the end of the article

\begin{abstract}
A modified fractional model for the magnetohydrodynamic (MHD) flow of a fluid is developed utilizing Atangana-Baleanu fractional derivative (ABFD). Natural convection and wall oscillation instigate the flow over a vertical plate positioned in a porous medium. The partial differential equations (PDEs) are transmuted to ordinary differential equations (ODEs). The Laplace transform method with its inversion is employed to accomplish the exact solutions of momentum and heat equations. The final solution is expressed in terms of gamma function, modified Bessel function, and Mittag-Leffler function. The previous definitions Caputo fractional and Riemann-Liouville are rarely used by the researchers now due to their limitations. The newly introduced ABFD has got significance nowadays due to its nonlocal and nonsingular kernel. This work focuses on the oscillating boundary conditions for the viscous model in terms of ABFD. The influence of involved parameters is interpreted through plots. The velocity profile is an increasing function of fractional parameter and jumps for a higher Grashof number due to buoyancy push. Furthermore, the Atangana-Baleanu (AB) model is compared with the ordinary derivative model for limiting case and analyzed in detail. It is noted that the ordinary fluid flows faster compared to the fractional fluid.
\end{abstract}

Keywords: Fluid flow; Natural convection; Atangana-Baleanu fractional derivative; Laplace transform method

\section{Springer}

\section{Introduction}

The literature of natural convection is sufficiently rich for magnetohydrodynamic oscillatory flow with classical fluid models. These natural problems related to the engineering and sciences lack the memory effect description. The fractional derivatives can describe memory effects, rheology, viscoelastic effect, diffusive transport, and fluid flow. In sciences and engineering, such as fluid mechanics, biomedical engineering, earthquake engineering, chemical engineering, and cooling processes in industries, many problems are modeled in fractional differential equations. Finding an operator that can physically describe this fact was complicated. Several scientists proposed that fractional derivatives can fully describe the memory effects, but the time and space components are the vital factors in implementing this idea. Various real-world problems follow three mathematical functions, i.e., the power law function, the exponential decay function, and the generalized Mittag-

(c) The Author(s) 2020. This article is licensed under a Creative Commons Attribution 4.0 International License, which permits use sharing, adaptation, distribution and reproduction in any medium or format, as long as you give appropriate credit to the original author(s) and the source, provide a link to the Creative Commons licence, and indicate if changes were made. The images or other third party material in this article are included in the article's Creative Commons licence, unless indicated otherwise in a credit line to the material. If material is not included in the article's Creative Commons licence and your intended use is not permitted by statutory regulation or exceeds the permitted use, you will need to obtain permission directly from the copyright holder. To view a copy of this licence, visit http://creativecommons.org/licenses/by/4.0/. 
Leffler function [1]. These mentioned functions are the basis for many mathematical definitions of the fractional differential operators such as Caputo and Riemann-Liouville fractional derivatives based on the power law functions $[1,2]$. These two definitions have been followed by researchers, but they have a limitation that the Caputo fractional derivative has a singular kernel and the derivative of a constant is not zero Riemann-Liouville fractional derivative [3]. These limitations can be overcome by Caputo-Fabrizio fractional derivative but due to the locality in its kernel this definition also exhibit limitations $[4,5]$. Shedding some light on these two definitions, some quality literature work is worth discussing here to understand their importance. Baleanu and Fernandez [6] established a new formula consisting of Mittag-Leffler kernel in the form of a series of Riemann-Liouville fractional integrals. Several varieties of fractional definitions can be found in the literature. Fernandez et al. [7] expressed the Prabhakar fractional model and its generalized forms as a series of Riemann-Liouville integrals. In a recent study, a generalization for the existing definitions of fractional derivatives and integrals was established by Fernandez et al. [8]. They introduced an integral operator with a general kernel and expressed it as an infinite series of the Riemann-Liouville integrals. Recently, Jarad and Abdeljawad [9] established generalized noninteger derivatives and the Laplace transform to solve dynamical systems in the fractional derivatives frame. Jarad et al. [10] developed a new class of fractional operators in the Reimann-Liouville and Caputo sense. Some relevant references to the fractional integrals and their applications can be found in [11-15] and the references therein.

Azhar et al. [16] used the Caputo-Fabrizio time fractional derivative to the problem of nanofluid flow over a moving vertical plate. Fetecau et al. [17] conducted an analysis on the flow of a nanofluid over an isothermal plate Caputo-time fractional derivative. The flow of a generalized second grade fluid between parallel plates with Riemann-Liouville fractional derivative model was investigated by Wenchang and Mingyu [18]. They acquired the exact analytical solution using the Laplace transform and the Fourier transform. The flow of a second order fluid induced by a plate moving impulsively with fractional anomalous diffusion was investigated by Mingyu and Wenchang [19]. The Rayleigh-Stokes problem for a fractional second grade fluid was studied by Shen et al. [20]. The fractional Laplace transform and Fourier sine transform were employed to obtain the exact solution. Exact analytical solution unsteady flow of a generalized Maxwell fluid between two circular cylinders was determined via Laplace and Hankel transforms [21]. Recently Shen et al. [22] studied a fractional Maxwell viscoelastic nanofluid for various particle shapes. Caputo time fractional derivative was implemented by Zhang et al. [23] to acquire the numerical and analytical solutions for the problem of 2D flow of Maxwell fluid under variable pressure gradient. They used the separation of variables method to acquire analytical solution while for numerical solution the finite difference method was used. Aman et al. [24] studied a fractional Maxwell fluid for exact analytical solution with second order slip.

Now to tackle the above discussed issue of local kernel, Atangana and Baleanu [25] proposed a new fractional derivative with nonlocal and nonsingular kernels, which fulfills all the mathematical principles related to fractional calculus. The ABFD definition is based on the Mittag-Leffler function and promises an improved description of dynamics of the system with the memory effects [26]. ABFD in the Caputo sense is used to investigate the model of vertical transmission and to cure a vector-borne disease [27]. They found the model very effective in curing the disease with a decrease in transmission rate. Very limited 
research can be found on fluid mechanics problems reporting fluid flow, heat transfer, and other world problems in applied mathematics and mathematical physics. Gomez-Aguilar et al. [28] presented a three-dimensional cancer model using Caputo-Fabrizio-Caputo and Atangana-Baleanu-Caputo fractional order derivatives. This model was proposed to study the evolution of cancer growth by the interactions of tumor cells with healthy cells and activated immune system cells. A scheme via the Laplace transform, the SumuduPicard integration method, and the Adams-Moulton rule was used to acquire the solutions.

Jan et al. [29] determined the solution for Brinkman type nanofluid using an AtanganaBaleanu fractional model. Owolabi and Atangana [30] analyzed the numerical simulation of an Adams-Bashforth scheme using Atangana-Baleanu-Caputo fractional derivatives. Saad et al. [31] established numerical solutions for the fractional Fisher type equation using ABFD. They employed a spectral collocation method based on Chebyshev approximations. In this research work, the spectral collocation method was implemented for the first time to solve nonlinear equation with Atangana-Baleanu derivatives. The logarithmic-KdV equation with the $\mathrm{AB}$ fractional operator and Mittag-Leffler type kernel was investigated by Mustafa et al. [32] using the numerical iterative method. Some plenteous literature regarding $\mathrm{ABFD}$, their analytical solution, and applications can be found in [33-36] and the references therein. A few previous studies with ABFD are referenced in $[34,35,37,38]$, but mostly they are focused on non-Newtonian fluids, nanofluids, or some are formulated with constant wall velocity. The research work carried out on nanofluids connects to an entirely different formulation and outcomes as they emphasize more the thermophysical properties of the nanoparticles and fluid.

Recently research with huge practical significance was carried out on human immunodeficiency virus-tuberculosis (HIV-TB) coinfected model using ABFD and the MittagLeffler law by Khan et al. [39]. They carried out the simulations for the existence, uniqueness of solution, and Hyers-Ulam (HU) stability. Another interesting research was done using ABFD for human blood flow nanofluids by Khan [40]. Their target was the investigation of nanofluids with channel flow in terms of the ABFD model and via the Laplace transform technique. They considered constant wall velocity at the boundaries. There is a gap for these combinations of studies in terms of oscillating boundary conditions for the ABFD model. Thus, the proposed study will focus on the ABFD model of an oscillatory Newtonian fluid flow with magnetic and radiative heat flux.

The literature reveals the importance of ABFD in the fluid dynamics domain. There is limited literature that discusses the free convection fluid flow using an Atangana-Baleanu derivative model for oscillatory flow; therefore the authors are motivated to conduct this research. Exact solutions are to be established for the problem of natural convection flow of fluid over a vertical plate using ABFD. The analytical solution is traced out via the Laplace transform method with its inversion. The solution is particularized for limiting case, i.e., for an ordinary fluid model by making $\alpha=1$. Finally, a brief graphical interpretation has been made for various parameters and comparison with the ordinary fluid model.

The structure of the paper is arranged as follows. In Sect. 2 we derive the mathematical ABFD model for the problem under consideration. The analytical solution is given in Sect. 3, we also recover the limiting case here. The acquired results are explained briefly in Sect. 4 followed by the conclusion of the paper in Sect. 5 . 


\section{Mathematical framework}

Consider unsteady flow of a fluid over a vertical plate with heat flux taken into consideration. A uniform magnetic field is employed perpendicular to the flow of the fluid. The flow is induced by the natural buoyancy force and heat flux $q_{r}$ on the plate. The temperature on the surface of the plate is $T_{w}$, while $T_{\infty}$ is the temperature far away from the surface. The plate starts oscillations at time $t>0$. The physical interpretation of the flow is shown in Fig. 1. The governing equations of momentum and energy are given as follows:

$$
\begin{aligned}
& \rho_{f} \cdot \frac{\partial u}{\partial t}=\mu \frac{\partial^{2} u}{\partial y^{2}}-\left(\frac{\mu}{k_{1}}+B_{0}^{2} \sigma_{f}\right) u+\mathrm{g} \beta_{f} \rho_{f}\left(T-T_{\infty}\right), \\
& \left(\rho c_{p}\right)_{f} \cdot \frac{\partial T}{\partial t}=k_{f} \frac{\partial^{2} T}{\partial y^{2}}-\frac{\partial q_{r}}{\partial y} .
\end{aligned}
$$

Here, $T$ and $u$ denote temperature and velocity, $\rho_{f}, \mu, k_{1}, B_{0}^{2}, \sigma_{f}, \beta_{f}, k_{f}, q_{r}$ are the density, dynamic viscosity, porosity parameter, magnetic parameter, electrical conductivity, thermal expansion coefficient, thermal conductivity, and radiative flux parameter. The radiative heat flux is defined as in [16, 24]:

$$
-\frac{\partial q_{r}}{\partial y}=4 \alpha\left(T-T_{0}\right)
$$

The boundary conditions are

$$
\begin{array}{lc}
T(y, 0)=T_{\infty}, \quad T(0, t)=T_{w}, \quad T(\infty, t)=T_{\infty}, \\
u(y, 0)=0, \quad u(0, t)=U_{0} \cos \omega t, \quad u(\infty, t)=0,
\end{array}
$$

where $U_{0}$ is the amplitude and $\omega$ is the frequency of oscillations.

The similarity variables are as follows:

$$
y^{*}=\frac{y U_{0}}{v}, \quad u^{*}=\frac{u}{U_{0}}, \quad t^{*}=\frac{t U_{0}^{2}}{v}, \quad \theta=\frac{T-T_{\infty}}{T_{w}-T_{\infty}} .
$$

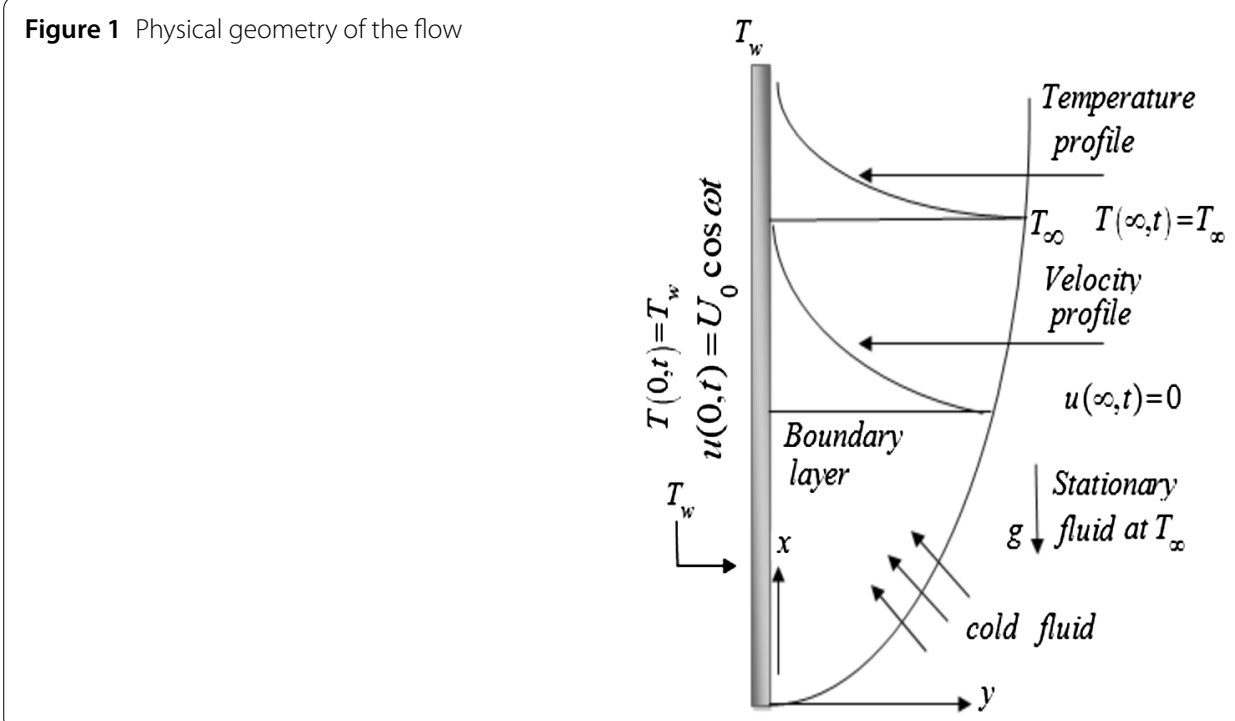


Transforming Eqs. (1)-(4) to dimensionless PDEs using Eq. (5), we get (asterisk" is omitted for convenience):

$$
\begin{aligned}
& \frac{\partial u}{\partial t}=\frac{\partial^{2} u}{\partial y^{2}}-(k+M) u+G r_{T} \theta \\
& \operatorname{Pr} \cdot \frac{\partial \theta}{\partial t}=\frac{\partial^{2} \theta}{\partial y^{2}}+N^{2} \theta
\end{aligned}
$$

with the boundary conditions

$$
\begin{aligned}
& \theta(y, 0)=0, \quad \theta(0, t)=1, \quad \theta(\infty, t)=0, \\
& u(y, 0)=0, \quad u(0, t)=\cos \omega t, \quad u(\infty, t)=0,
\end{aligned}
$$

where

$$
\begin{aligned}
& k=\frac{v^{2}}{k_{1} U_{0}^{2}}, \quad M=\frac{v B_{0}^{2} \sigma_{f}}{\rho_{f} U_{0}^{2}}, \quad N^{2}=\frac{4 \alpha^{2} v^{2}}{k_{f} U_{0}^{2}}, \\
& G r_{T}=\frac{g \beta_{f} v\left(T_{w}-T_{\infty}\right)}{U_{0}^{3}}, \quad \operatorname{Pr}=\frac{\left(\rho c_{p}\right)_{f} v}{k_{f}},
\end{aligned}
$$

where $k, M, G r_{T}, \operatorname{Pr}, N, v, \alpha$ denote the porosity, magnetic, Grashof number, Prandtl number, radiation parameter, kinematic viscosity, and mean radiation absorption coefficient respectively. Here $v=\frac{\mu}{\rho_{f}}$. The ABFD model of the present problem is given by

$$
\begin{aligned}
& { }^{\mathrm{AB}} D_{t}^{\alpha} u=\frac{\partial^{2} u}{\partial y^{2}}-(k+M) u+G r_{T} \theta, \\
& \operatorname{Pr} \cdot{ }^{\mathrm{AB}} D_{t}^{\alpha} \theta=\frac{\partial^{2} \theta}{\partial y^{2}}+N^{2} \theta .
\end{aligned}
$$

Atangana and Baleanu fractional derivative [25] is defined as

$$
{ }^{\mathrm{AB}} D_{t}^{\alpha} f(y, t)=\frac{N(\alpha)}{1-\alpha} \int_{0}^{t} E_{\alpha}\left(-\alpha \frac{(t-\tau)^{\alpha}}{1-\alpha}\right) \frac{\partial f(y, \tau)}{\partial \tau} d \tau,
$$

where $N(\alpha)$ is a normalization function such that $N(0)=N(1)=1$ and $E_{\alpha}(z)=\sum_{k=0}^{\infty} \frac{z^{k}}{\Gamma(\alpha k+1)}$ is a Mittag-Leffler function [41]. The Laplace transform of Eq. (13) is given as follows:

$$
\mathcal{L}\left\{{ }^{\mathrm{AB}} D_{t}^{\alpha} f(\mathrm{t})\right\}=\frac{q^{\alpha} \mathcal{L}\{f(t)\}-q^{\alpha-1} f(0)}{q^{\alpha}(1-\alpha)+\alpha},
$$

where $q$ denotes the Laplace transform operator.

\section{Analytical solution}

Applying the Laplace transform to Eqs. (8)-(12) and utilizing the definition given in Eq. (14), we get

$$
\frac{q^{\alpha} \bar{u}}{q^{\alpha}(1-\alpha)+\alpha}=\frac{\partial^{2} \bar{u}}{\partial y^{2}}-(k+M) \bar{u}+G r_{T} \bar{\theta},
$$




$$
\begin{aligned}
& \frac{\operatorname{Pr} q^{\alpha} \bar{\theta}}{q^{\alpha}(1-\alpha)+\alpha}=\frac{\partial^{2} \bar{\theta}}{\partial y^{2}}+N^{2} \bar{\theta}, \\
& \bar{\theta}(0, q)=\frac{1}{q}, \quad \bar{\theta}(\infty, 0)=0 \\
& \bar{u}(0, q)=\frac{q}{q^{2}+\omega^{2}}, \quad \bar{u}(\infty, 0)=0 .
\end{aligned}
$$

Thus

$$
\begin{aligned}
\bar{\theta}(y, q)= & \left(\frac{1}{q^{1-\alpha}}\right)\left(\frac{1}{q^{\alpha}}\right) \mathrm{e}^{-\mathrm{y} \sqrt{b_{3}} \sqrt{\frac{q^{\alpha}+b_{2}}{q^{\alpha}+b_{1}}}} \\
\bar{u}(y, q)= & \left(\frac{q}{q^{2}+\omega^{2}}\right) \mathrm{e}^{-\mathrm{y} \sqrt{b_{4}} \sqrt{\frac{q^{\alpha}+b_{6}}{q^{\alpha}+b_{1}}}}+\frac{G r_{T}}{b_{7}}\left(\frac{q^{\alpha}+b_{1}}{b_{3}\left(q^{\alpha}+b_{2}\right)-\left(b_{4} q^{\alpha}+b_{5}\right)}\right) \mathrm{e}^{-\mathrm{y} \sqrt{b_{4}} \sqrt{\frac{q^{\alpha}+b_{6}}{q^{\alpha}+b_{1}}}} \\
& -\frac{G r_{T}}{b_{7}}\left(\frac{q^{\alpha}+b_{1}}{b_{3}\left(q^{\alpha}+b_{2}\right)-\left(b_{4} q^{\alpha}+b_{5}\right)}\right) \mathrm{e}^{-\mathrm{y} \sqrt{b_{3}} \sqrt{\frac{q^{\alpha}+b_{2}}{q^{\alpha}+b_{1}}}}
\end{aligned}
$$

where

$$
\begin{aligned}
& b_{0}=\frac{1}{(1-\alpha)}, \quad b_{1}=b_{0} \alpha, \quad b_{2}=\frac{b_{1} N^{2}}{b_{3}}, \quad b_{3}=b_{0} \operatorname{Pr}+N^{2}, \\
& b_{4}=\left(b_{0}+k+M\right), \quad b_{5}=b_{1}(k+M), \quad b_{6}=\frac{b_{5}}{b_{4}}, \\
& b_{7}=b_{3}-b_{4}, \quad b_{8}=\frac{b_{2} b_{3}-b_{4} b_{5}}{b_{7}} .
\end{aligned}
$$

Equations (19) and (20) can be written as follows:

$$
\begin{aligned}
\bar{\theta}(y, q)= & \bar{h}(q, \alpha) \bar{\Phi}\left(y \sqrt{b_{3}}, q ; 0, b_{2}, b_{1}\right), \\
\bar{u}(y, q)= & \left(\frac{q}{q^{2}+\omega^{2}}\right) \bar{\Phi} 1\left(y \sqrt{b_{4}}, q ; 0, b_{6}, b_{1}\right)+\frac{G r_{T}}{b_{7}}\left(h(q, \alpha)+\left(b_{1}-b_{8}\right)\left(\frac{q^{\alpha-1}}{q^{\alpha}+b_{8}}\right)\right) \\
& \times\left[\bar{\Phi}\left(y \sqrt{b_{4}}, q ; 0, b_{6}, b_{1}\right)-\bar{\Phi}\left(y \sqrt{b_{3}}, q ; 0, b_{2}, b_{1}\right)\right],
\end{aligned}
$$

where

$$
\begin{aligned}
& \bar{h}(q, \alpha)=\left(\frac{1}{q^{1-\alpha}}\right), \quad \overline{\Phi 1}\left(y \sqrt{b_{4}}, q ; 0, b_{6}, b_{1}\right)=\mathrm{e}^{-\mathrm{y} \sqrt{b_{4}} \sqrt{\frac{q^{\alpha}+b_{6}}{q^{\alpha}+b_{1}}}} \\
& \bar{\Phi}\left(y \sqrt{b_{3}}, q ; 0, b_{2}, b_{1}\right)=\left(\left(\frac{1}{q^{\alpha}}\right) \mathrm{e}^{-\mathrm{y} \sqrt{b_{3}} \sqrt{\frac{q^{\alpha}+b_{2}}{q^{\alpha}+b_{1}}}}\right) \\
& \bar{\Phi}\left(y \sqrt{b_{4}}, q ; 0, b_{6}, b_{1}\right)=\left(\left(\frac{1}{q^{\alpha}}\right) \mathrm{e}^{-\mathrm{y} \sqrt{b_{4}} \sqrt{\frac{q^{\alpha}+b_{6}}{q^{\alpha}+b_{1}}}}\right) .
\end{aligned}
$$

Employing the inverse Laplace transform to Eqs. (22) and (23), utilizing the convolution theorem (A1) and Appendix (A2)-(A5), the final expression is obtained as follows:

$$
\theta(y, t)=\int_{0}^{t} h(t-s, \alpha) \Phi\left(y \sqrt{b_{3}}, s ; 0, b_{2}, b_{1}\right) d s
$$




$$
\begin{aligned}
u(y, t)= & \int_{0}^{t} \cos (\omega(t-s)) \Phi 1\left(y \sqrt{b_{4}}, s ; 0, b_{6}, b_{1}\right) d s \\
& +\frac{G r}{b_{7}}\left(\int_{0}^{t} h(t-s, \alpha) \Phi\left(y \sqrt{b_{4}}, s ; 0, b_{6}, b_{1}\right) d s\right. \\
& \left.+\left(b_{1}-b_{8}\right) \int_{0}^{t} E_{\alpha, 1}\left(-b_{8}(t-s)^{\alpha}\right) \Phi\left(y \sqrt{b_{4}}, s ; 0, b_{6}, b_{1}\right) d s\right) \\
& -\frac{G r}{b_{7}}\left(\int_{0}^{t} h(t-s, \alpha) \Phi\left(y \sqrt{b_{3}}, s ; 0, b_{2}, b_{1}\right) d s\right. \\
& \left.+\left(b_{1}-b_{8}\right) \int_{0}^{t} E_{\alpha, 1}\left(-b_{8}(t-s)^{\alpha}\right) \Phi\left(y \sqrt{b_{3}}, s ; 0, b_{2}, b_{1}\right) d s\right),
\end{aligned}
$$

where

$$
\begin{aligned}
& \Phi\left(y \sqrt{b_{3}}, t ; 0, b_{2}, b_{1}\right) \\
& =\frac{1}{\pi} \int_{0}^{\infty} \int_{0}^{\infty} \psi\left(y \sqrt{b_{3}}, u ; 0, b_{2}, b_{1}\right) \\
& \times \exp \left(-\tau-r-u r^{\alpha} \cos \alpha \pi\right)\left(u r^{\alpha} \sin \alpha \pi\right) d r d u, \\
& \Phi\left(y \sqrt{b_{4}}, t ; 0, b_{6}, b_{1}\right) \\
& =\frac{1}{\pi} \int_{0}^{\infty} \int_{0}^{\infty} \psi\left(y \sqrt{b_{4}}, u ; 0, b_{6}, b_{1}\right) \\
& \times \exp \left(-\tau-r-u r^{\alpha} \cos \alpha \pi\right)\left(u r^{\alpha} \sin \alpha \pi\right) d r d u, \\
& \overline{\Phi 1}\left(y \sqrt{b_{4}}, t ; 0, b_{6}, b_{1}\right) \\
& =\frac{1}{\pi} \int_{0}^{\infty} \int_{0}^{\infty} \psi\left(y \sqrt{b_{4}}, u ; 0, b_{6}, b_{1}\right) \\
& \times \exp \left(-t r-u r^{\alpha} \cos \alpha \pi\right) \sin \left(u r^{\alpha} \sin \alpha \pi\right) d r d u, \\
& \psi\left(y \sqrt{b_{3}}, t ; 0, b_{2}, b_{1}\right) \\
& =e^{-y \sqrt{b_{3}}}-\frac{y \sqrt{b_{2}-b_{1}}}{2 \pi} \\
& \times \int_{0}^{\infty} \int_{0}^{t} \frac{1}{\sqrt{t}} e^{\left(-b_{1} t-\frac{y^{2}}{4 u}-u\right)} I_{1}\left(2 \sqrt{\left(b_{2}-b_{1}\right) u t}\right) d t d u \\
& E_{\alpha, 1}\left(-b_{8} t^{\alpha}\right)=\sum_{k=0}^{\infty} \frac{\left(-b_{8}(t)^{\alpha}\right)^{k}}{\Gamma(\alpha k+1)}, \quad I_{n}(x)=\sum_{k=0}^{\infty} \frac{(-1)^{k}}{k !(n+k) !}\left(\frac{x}{2}\right)^{n+2 k} ; \quad n \geq 0 .
\end{aligned}
$$

Here $I_{1}(x)$ is a Bessel function of the first kind.

\subsection{Limiting case}

Taking $\alpha=1$ in Eq. (14), we acquire the limiting case by recovering the solution for the classical model of the present problem:

$$
\lim _{\alpha \rightarrow 1} D_{t}^{\alpha} \bar{f}(y, t)=\lim _{\alpha \rightarrow 1} \mathcal{L}^{-1}\left\{\frac{q^{\alpha} \mathcal{L}\{f(y, t)\}-q^{\alpha-1} f(y, 0)}{q^{\alpha}(1-\alpha)+\alpha}\right\}
$$




$$
\begin{aligned}
& =\mathcal{L}^{-1}\left\{\lim _{\alpha \rightarrow 1} \frac{q^{\alpha} \bar{f}(y, q)-q^{\alpha-1} f(y, 0)}{q^{\alpha}(1-\alpha)+\alpha}\right\} \\
& =\mathcal{L}^{-1}\{q \bar{f}(y, q)-f(y, 0)\} .
\end{aligned}
$$

Thus, the solution we recovered is given as follows:

$$
\begin{aligned}
\theta(y, t)= & \frac{1}{2}\left[e^{-y\left(\operatorname{Pr} \sqrt{-N^{2}}\right)} \cdot \operatorname{erfc}\left(\frac{y \sqrt{\operatorname{Pr}}}{2 \sqrt{t}}-\sqrt{\left(-\frac{N^{2}}{\operatorname{Pr}}\right) t}\right)\right. \\
& \left.+e^{y\left(\operatorname{Pr} \sqrt{-N^{2}}\right)} \cdot \operatorname{erfc}\left(\frac{y \sqrt{\operatorname{Pr}}}{2 \sqrt{t}}+\sqrt{\left(-\frac{N^{2}}{\operatorname{Pr}}\right) t}\right)\right], \\
u(y, t)= & \int_{0}^{t} \cos (\omega(t-s)) \Phi 1\left(y \sqrt{b_{4}}, s ; 0, b_{6}, b_{1}\right) d s \\
& +\frac{G r}{b_{7}}\left(\int_{0}^{t} h(t-s, \alpha) \Phi\left(y \sqrt{b_{4}}, s ; 0, b_{6}, b_{1}\right) d s\right. \\
& \left.+\left(b_{1}-b_{8}\right) \int_{0}^{t} E_{\alpha, 1}\left(-b_{8}(t-s)^{\alpha}\right) \Phi\left(y \sqrt{b_{4}}, s ; 0, b_{6}, b_{1}\right) d s\right) .
\end{aligned}
$$

\section{Results and discussion}

This section highlights the principal features and acquired results for the analysis of oscillatory flow of a fluid under free convection and radiation. The analytical solution for the present problem in terms of the Atangana-Baleanu fractional model has been established via the Laplace transform method. Gamma function, modified Bessel function, and Mittag-Leffler functions are used to express the final general solution for momentum and heat equation. The flow and heat are analyzed for the influence of the parameters such as fractional parameter, radiation parameter, magnetic parameter, porosity parameter, and Grashof number. At the end, a comparison analysis has been accomplished between the fractional and ordinary models. The brief findings in the present analysis are stated below.

Figure 2(a)-(b) shows the temperature field at $t=2$, with the variation of fractional parameter $\alpha$. The maximizing values of fractional parameter $\alpha$ cause a rise in the temper-

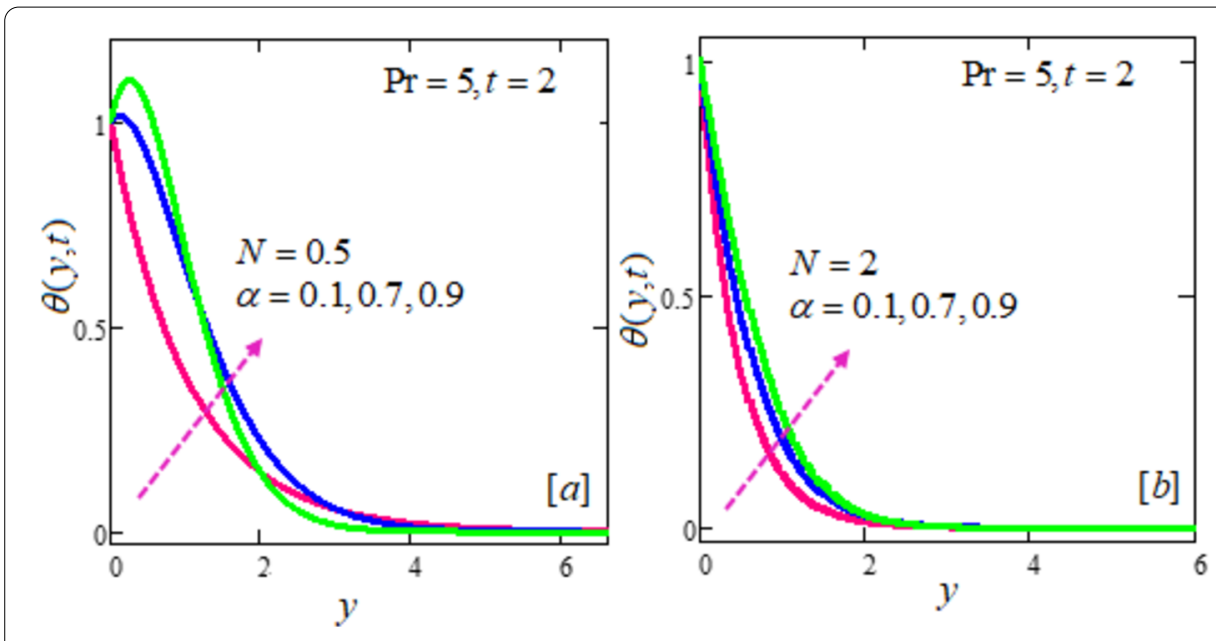

Figure 2 Temperature profile variation with fractional parameter $\alpha$ and radiation parameter $N$ 

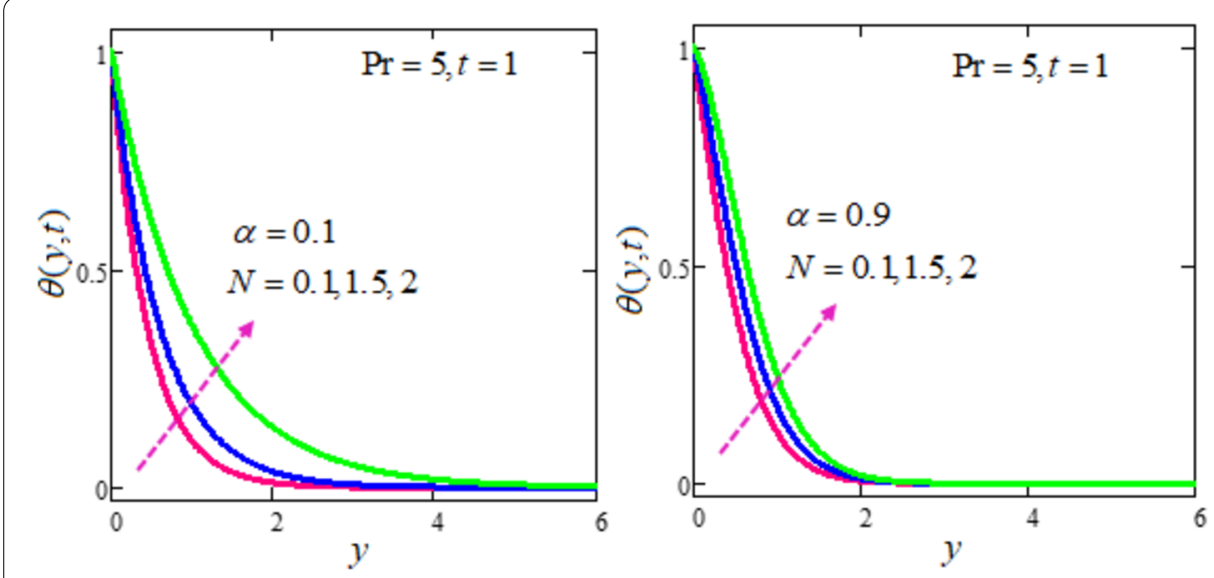

Figure 3 Temperature profile variation with radiation parameter $N$ and fractional parameter $\alpha$
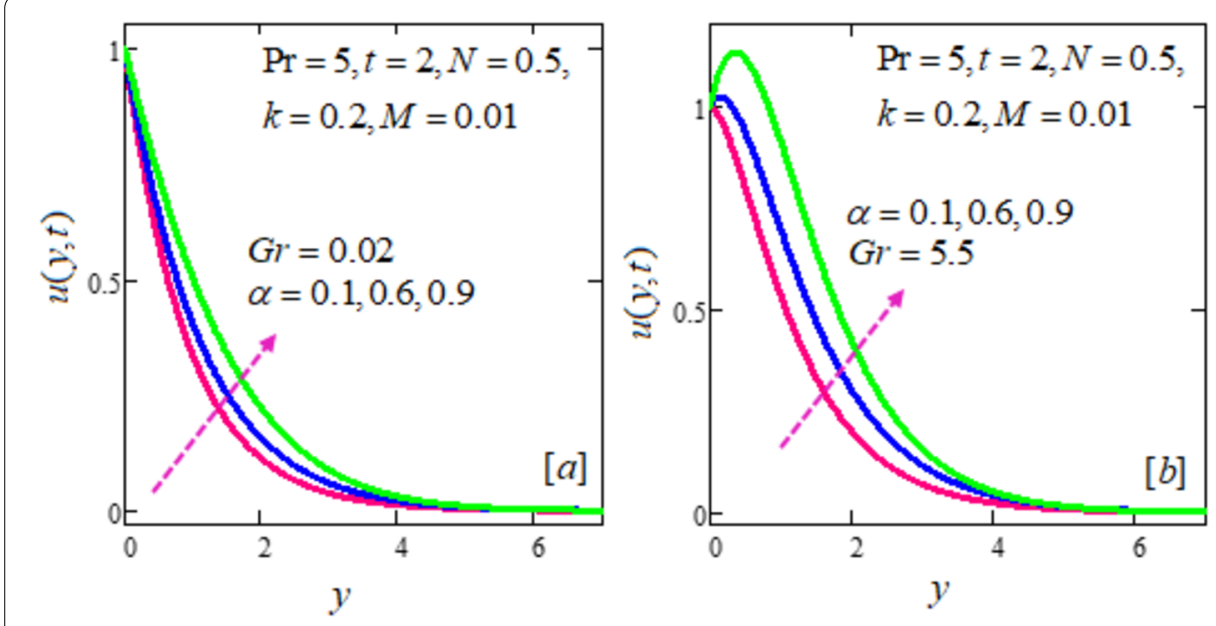

Figure 4 Velocity profile variation with fractional parameter $\alpha$ and Grashof number Gr

ature profile for $\alpha=0.1,0.7,0.9$. In Fig. 2(a), the temperature profile for $N=0.5$ is low, while in Fig. 2(b) the temperature shows a unique rising behavior at $\alpha=0.7$ and $\alpha=0.9$ for greater radiation parameter, i.e., $N=2$. Figure 3(a)-(b) depicts the temperature variation under the effect of radiation parameter $N$. The results are plotted at two different values of $\alpha$. The temperature profile of the fluid increases significantly for $N=0.1,1.5,2$ in Figs. 3(a) and 3(b) at $\alpha=0.1$ and $\alpha=0.9$. The temperature rise is more significant at the higher value of fractional parameter for $\operatorname{Pr}=5, t=1$.

Figure 4(a)-(b) shows that the fluid velocity increases when the fractional parameter value varies as $\alpha=0.1,0.2,0.3$, while other parameters are kept constant, i.e., $N=0.5$, $\operatorname{Pr}=5, M=0.01, k=0.2$. The results are acquired at two different values of the Grashof number $G r$ to interpret the simultaneous influence of the Grashof number and the fractional parameter. For low buoyancy forces, $G r=0.02$, the flow gradually rises in Fig. 4(a) with variation of $\alpha$. A noteworthy increasing behavior can be seen for $G r=5.5 \mathrm{in} \mathrm{Fig.} \mathrm{4(b)}$ due to a huge push of buoyancy forces. All these observations are accomplished at $t=2$. 


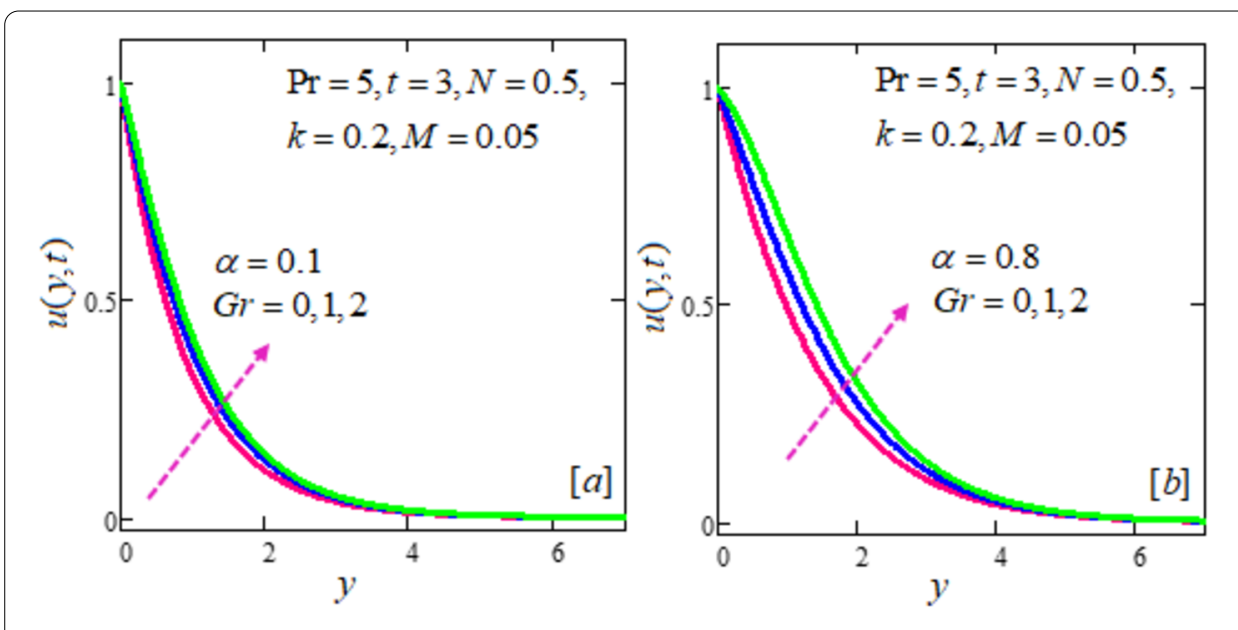

Figure 5 Velocity profile variation with Grashof number Gr and fractional parameter $\alpha$

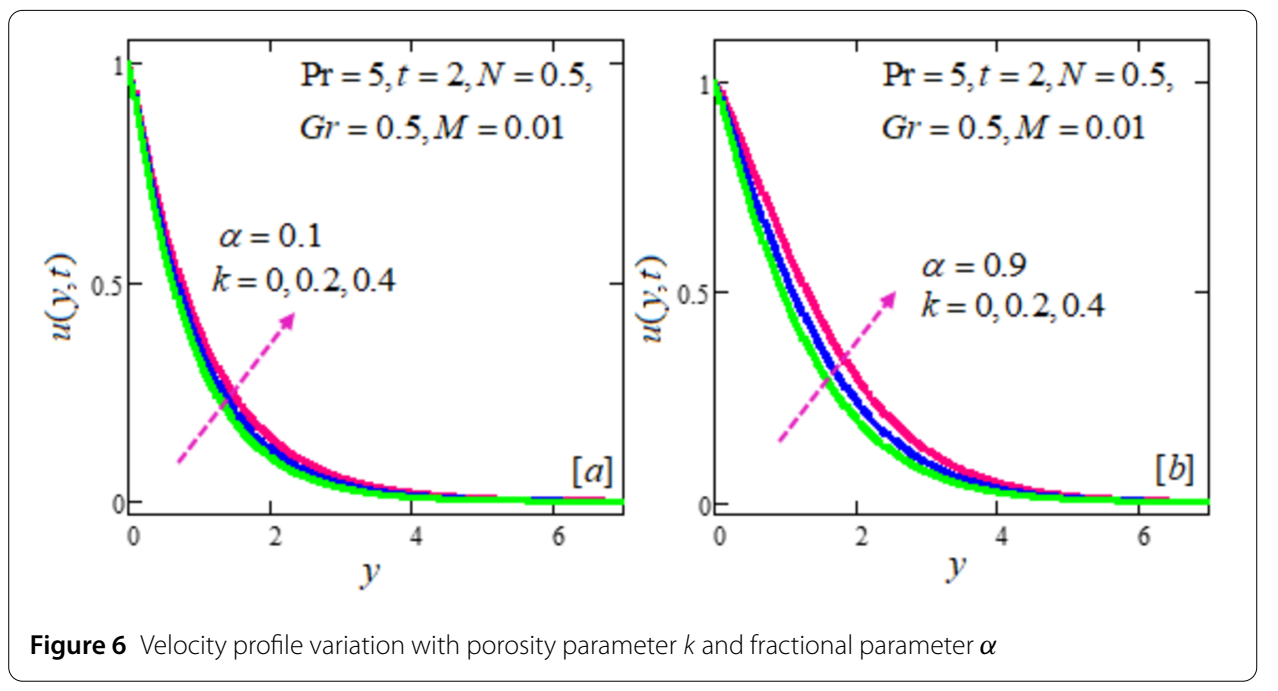

In Fig. 5(a)-(b), the fluid velocity increases with the varying Grashof number $\mathrm{Gr}=0,1,2$ for other constant parameters, i.e., $N=0.5, t=3, \operatorname{Pr}=5, M=0.05, k=0.2$. Here, the results are plotted at $\alpha=0.1$ and $\alpha=0.8$ to examine the parallel influence of $\alpha$ with $G r$. At some small fractional parameter $\alpha=0.1$, the flow variation is not much significant in Fig. 5(a), while at a value $\alpha=0.8$ there is a considerable rise in Fig. 5(b) with variation of $\alpha$. The variation in velocity is remarkable at a greater value of the fractional parameter for the same variation in the Grashof number.

Figure 6(a)-(b) shows that the fluid velocity increases with the increasing porosity parameter $k=0,0.2,0.4$, while other parameters are constant, i.e., $N=0.5, \operatorname{Pr}=5, M=0.01$, $G r=0.5$. For small fractional parameter $\alpha=0.1$, the flow gradually rises in Fig. 6(a) with variation of $k$. The increasing behavior for $\alpha=0.9 \mathrm{in} \mathrm{Fig.} \mathrm{6(b)} \mathrm{is} \mathrm{considerable} \mathrm{compared} \mathrm{to}$ that in case of smaller $\alpha$ due to a huge push of buoyancy forces. All these observations are carried out at $t=2$.

In Fig. 7(a)-(b), the fluid velocity is observed at two different values of $\alpha$, i.e., $\alpha=0.2, \alpha=$ 0.9 , for the variation of magnetic parameter $M=0.01,0.2,0.4$. The remaining parameters are considered constant for this observation, i.e., $N=0.5, \operatorname{Pr}=5, t=2, k=0.2, G r=0.5$. 

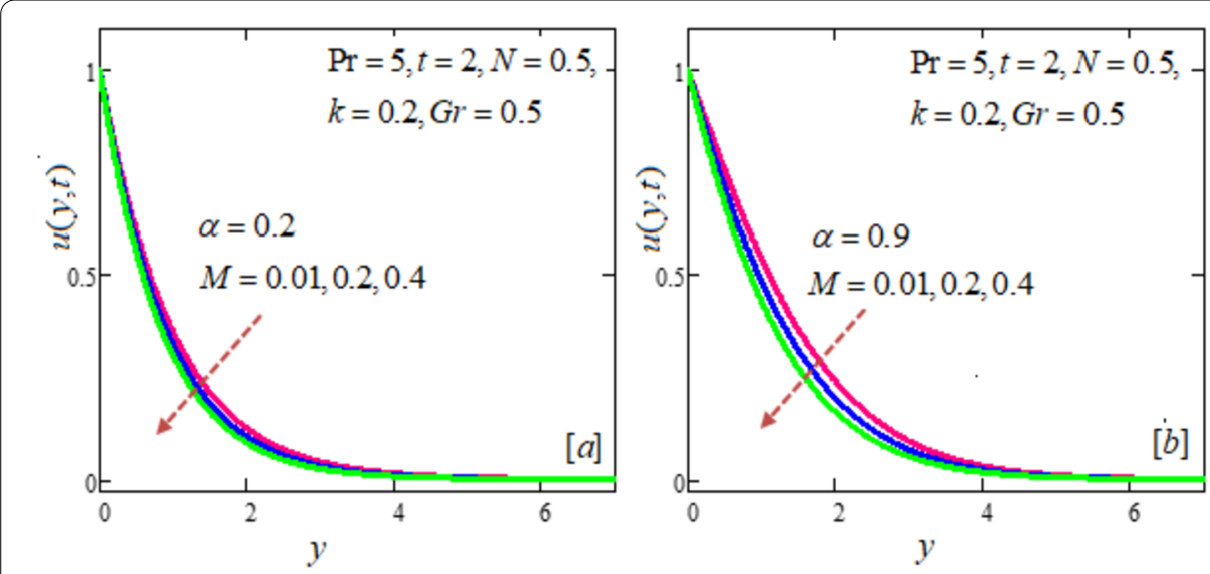

Figure 7 Velocity profile variation with magnetic parameter $M$ and fractional parameter $\alpha$
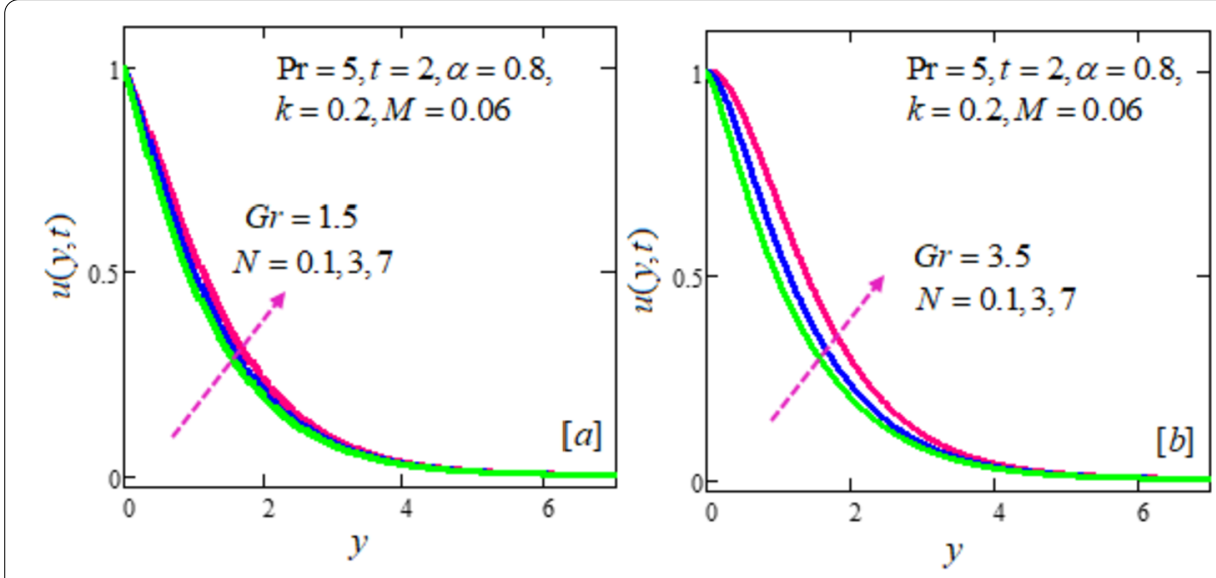

Figure 8 Velocity profile variation with radiation parameter $N$ and Grashof number $G r$

Fluid velocity tends to decrease gradually in Figs. 7(a) and 7(b), but the effect is more significant with higher velocity range in case of a greater value of $\alpha$.

In Fig. 8(a)-(b), the velocity profile is analyzed for two different values of the Grashof number, i.e., $G r=1.5, G r=3.5$, with the variation of radiation parameter $N=0.1,3,7$. The greater buoyancy forces lead to a significant variation in the velocity profile for various values of $N$, while for smaller value of $G r$, the velocity variation with radiation parameter is not much obvious [Figs. 8(a)-8(b)]. These inspections are carried out for constant values $M=0.06, \operatorname{Pr}=5, t=2, k=0.2, \alpha=0.8$. It is obvious from all the results that for greater values of $G r$ and $\alpha$ the variation with any other parameter becomes significant and higher compared to that of smaller values of $G r$ and $\alpha$. The higher buoyancy forces push the fluid to flow faster.

In Fig. 9, the temperature profile of the current fractional problem is compared with the ordinary model of the problem by making $\alpha=1$ in the heat equation (Eq. (2)). The deviation at some points is clear at $t=1$ with constant parameters $\operatorname{Pr}=5, N=1.5$. It is worth noting that both ordinary and fractional models have qualitatively identical behavior of fluid temperature. Figure 10 depicts the comparison for velocity profile of fractional derivative 


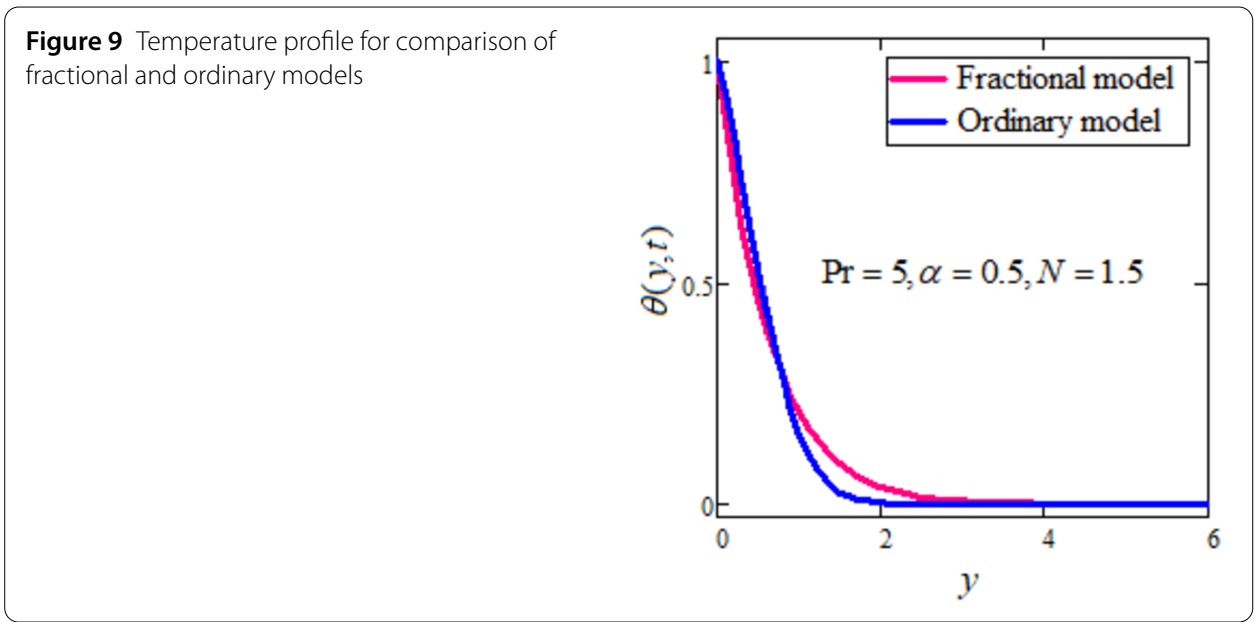

Figure 10 Velocity profile for comparison of fractional and ordinary models

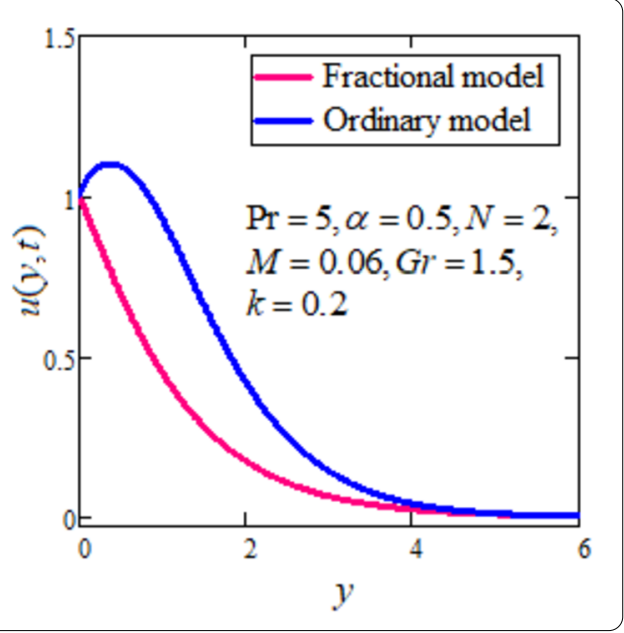

Table 1 Numerical values of $\theta(y, t)$ and $u(y, t)$ for fractional and ordinary fluid models

\begin{tabular}{lllll}
\hline $\begin{array}{l}\text { Distance from } \\
\text { plate " } y^{\prime \prime}\end{array}$ & $\begin{array}{l}\theta(y, t) \text { for } \\
\text { fractional fluid }\end{array}$ & $\begin{array}{l}\theta(y, t) \text { for } \\
\text { ordinary fluid }\end{array}$ & $\begin{array}{l}u(y, t) \text { for } \\
\text { fractional fluid }\end{array}$ & $\begin{array}{l}u(y, t) \text { for } \\
\text { ordinary fluid }\end{array}$ \\
\hline 0 & 1 & 1 & 0.999 & 0.999 \\
0.5 & 0.462 & 0.529 & 0.697 & 1.092 \\
1 & 0.205 & 0.155 & 0.451 & 0.929 \\
1.5 & 0.089 & 0.025 & 0.282 & 0.662 \\
2 & 0.037 & 0.0023 & 0.173 & 0.422 \\
\hline
\end{tabular}

$0>\alpha>1$ and the classical derivative model $\alpha=1$. The fluid with classical model has a unique and greater velocity than the fractional model. These results are accomplished at constant parameters, i.e., $M=0.06, \operatorname{Pr}=5, t=2, k=0.2, G r=1.5, N=2$. It is pointed out that the fractional fluid moves slower in comparison with the ordinary model. The numerical values for fractional and ordinary fluid models are depicted in Table 1, corresponding to the results in Figs. 9-10. These numerical values are evaluated for different distances of fluid from the plate, i.e., $0 \leq y \leq 2$. In consistency with these plots, the numerical values signify the abrupt changes when $\alpha=1$, i.e., the fractional fluid model is replaced by the ordinary one. For the fractional fluid, the highest value of velocity $u(y, t)$ is 0.422 , while for the ordinary fluid it is 0.173 . 


\section{Conclusion}

In this section, the concluding remarks for the analysis of a fluid under free convection and radiation are emphasized. The ABFD model has been extended for oscillatory flow and heat transfer model for which the analytical solution has been established in terms of Mittag-Leffler function via the attractive technique "Laplace transform method". Limiting case is recovered for an ordinary fluid when fractional parameter is taken to be one, i.e., $\alpha=1$. The main outcomes and concluding remarks are given as follows. Fluid velocity increases with increasing effect of the radiation parameter. But for the same constant parameters and varying radiation parameter, the velocity acquires a significant increase for greater value of the Grashof number. Fluid flows faster when the value of porosity parameter increases. The more permeable the medium is, the faster the fluid flows. The maximizing range of fractional parameter gives a unique jump to the velocity profile when the Grashof number is as high as 5.5 or above. A similar behavior is found in case of temperature for greater value of the radiation parameter. For the ordinary fluid, the temperature profile has identical behavior to the fractional model. The velocity profile is higher for the ordinary fluid compared to the fractional fluid.

\section{Appendix}

$$
\begin{aligned}
& f(t) * \mathrm{~g}(y, t)=\int_{0}^{t} f(t-s) \mathrm{g}(y, s) d s, \\
& \mathcal{L}^{-1}\left(\frac{q^{-1}}{q^{\alpha}+c_{0}}\right)=t^{\alpha} E_{\alpha, \alpha+1}\left(-c_{0} t^{\alpha}\right) ; \quad q>0, \\
& \mathcal{L}^{-1}(\psi(y \sqrt{a}, q ; a, b, c)) \\
& =\psi(y \sqrt{a}, t ; a, b, c) \\
& =e^{-a t-y}-\frac{y \sqrt{b-c}}{2 \pi} \int_{0}^{\infty} \int_{0}^{t} \frac{e^{-a t}}{\sqrt{t}} e^{\left(a t-c t-\frac{y^{2}}{4 u}-u\right)} I_{1}(2 \sqrt{(b-c) u t}) d t d u, \\
& \Phi(y \sqrt{a}, t ; a, b, c) \\
& =\frac{1}{\pi} \int_{0}^{\infty} \int_{0}^{\infty} \psi(y \sqrt{a}, u ; a, b, c) \\
& \times \exp \left(-t r-u r^{\alpha} \cos \alpha \pi\right) \sin \left(u r^{\alpha} \sin \alpha \pi\right) d r d u, \\
& \mathcal{L}^{-1}(h(q, \alpha))=h(t, \alpha)=\left(\frac{1}{t^{\alpha} \Gamma(1-\alpha)}\right), \quad \mathcal{L}^{-1}\left(\frac{q}{q^{2}+a^{2}}\right)=\cos (a t) .
\end{aligned}
$$

\section{Acknowledgements}

Third author would like to acknowledge and express their gratitude to the United Arab Emirates University, Al-Ain, UAE for providing the financial support with Grant No: 31S363-UPAR (4) 2018. The second author would like to thank Prince Sultan University for funding this work through research group Nonlinear Analysis Methods in Applied Mathematics (NAMAM) group number RG-DES-2017-01-17.

Funding

RG-DES-2017-01-17.

Availability of data and materials

Not applicable. 
Conflict of interests

The authors declare no conflict of interests.

\section{Competing interests}

The authors declare that they have no competing interests.

\section{Authors' contributions}

All three authors contributed equally to this article. All authors read and approved the final manuscript.

\section{Author details}

'Department of Mathematical Sciences, UAE University, Al Ain, United Arab Emirates. ${ }^{2}$ Department of Mathematics and General Sciences, Prince Sultan University, Riyadh, Saudi Arabia. ${ }^{3}$ Department of Medical Research, China Medical University, Taichung, Taiwan. ${ }^{4}$ Department of Computer Science and Information Engineering, Asia University, Taichung, Taiwan.

\section{Publisher's Note}

Springer Nature remains neutral with regard to jurisdictional claims in published maps and institutional affiliations.

\section{Received: 8 May 2020 Accepted: 11 June 2020 Published online: 19 June 2020}

\section{References}

1. Caputo, M.: Linear models of dissipation whose $Q$ is almost frequency independent-II. Geophys. J. Int. 13(5), 529-539 (1967)

2. Atangana, A.: Derivative with a New Parameter. Theory, Methods and Applications. Academic Press, San Diego (2015)

3. Caputo, M., Fabrizio, M.: A new definition of fractional derivative without singular kernel. Prog. Fract. Differ. Appl. 1, 73-85 (2015). https://doi.org/10.12785/pfda/010201

4. Alkahtani, B.S.T., Koca, I., Atangana, A.: New numerical analysis of Riemann-Liouville time-fractional Schrodinger with power, exponential decay, and Mittag-Leffler laws. J. Nonlinear Sci. Appl. 10(8), 4231-4243 (2017)

5. Losada, J., Nieto, J.J.: Properties of a new fractional derivative without singular kernel. Prog. Fract. Differ. Appl. 1, 87-92 (2015). https://doi.org/10.12785/pfda/010202

6. Baleanu, D., Fernandez, A.: On some new properties of fractional derivatives with Mittag-Leffler kernel. Commun. Nonlinear Sci. Numer. Simul. 59, 444-462 (2018). https://doi.org/10.1016/j.cnsns.2017.12.003

7. Fernandez, A., Baleanu, D., Srivastava, H.M.: Series representations for fractional calculus operators involving generalised Mittag-Leffler functions. Commun. Nonlinear Sci. Numer. Simul. 67, 517-527 (2019). https://doi.org/10.1016/j.cnsns.2018.07.035

8. Fernandez, A., Özarslan, M.A., Baleanu, D.: On fractional calculus with general analytic kernels. Appl. Math. Comput. 354, 248-265 (2019). https://doi.org/10.1016/j.amc.2019.02.045

9. Jarad, F., Abdeljawad, T.: Generalized fractional derivatives and Laplace transform. Discrete Contin. Dyn. Syst., Ser. S, 13(3), 709-722 (2019). https://doi.org/10.3934/dcdss.2020039

10. Jarad, F., Ugurlu, E., Abdeljawad, T., Baleanu, D.: On a new class of fractional operators. Adv. Differ. Equ. 2017(1), 247 (2017). https://doi.org/10.1186/s13662-017-1306-z

11. Abdeljawad, T., Baleanu, D.: On fractional derivatives with generalized Mittag-Leffler kernels. Adv. Differ. Equ. 2018(1), 468 (2018). https://doi.org/10.1186/s13662-018-1914-2

12. Shah, K., Sarwar, M., Baleanu, D.: Study on Krasnoselskii's fixed point theorem for Caputo-Fabrizio fractional differential equations. Adv. Differ. Equ. 2020, 178 (2020). https://doi.org/10.1186/s13662-020-02624-x

13. Nazir, G., Shah, K., Alrabaiah, H., Khalil, H., Khan, R.A.: Fractional dynamical analysis of measles spread model under vaccination corresponding to nonsingular fractional order derivative. Adv. Differ. Equ. 2020, 171 (2020). https://doi.org/10.1186/s13662-020-02628-7

14. Yusuf, A., Inc, M., Aliyu, A.I., Baleanu, D.: Efficiency of the new fractional derivative with nonsingular Mittag-Leffler kernel to some nonlinear partial differential equations. Chaos Solitons Fractals 116, 220-226 (2018). https://doi.org/10.1016/j.chaos.2018.09.036

15. Shah, K., Jarad, F., Abdeljawad, T.: On a nonlinear fractional order model of dengue fever disease under Caputo-Fabrizio derivative. Alex. Eng. J. (2020). https://doi.org/10.1016/j.aej.2020.02.022

16. Azhar, W.A., Vieru, D., Fetecau, C.: Free convection flow of some fractional nanofluids over a moving vertical plate with uniform heat flux and heat source. Phys. Fluids 29(8), 082001 (2017). https://doi.org/10.1063/1.4996034

17. Fetecau, C., Vieru, D., Azhar, W.A.: Natural convection flow of fractional nanofluids over an isothermal vertical plate with thermal radiation. Appl. Sci. 7(3), 247 (2017). https://doi.org/10.3390/app7030247

18. Wenchang, T., Mingyu, X.: Unsteady flows of a generalized second grade fluid with the fractional derivative model between two parallel plates. Acta Mech. Sin. 20,471-476 (2004). https://doi.org/10.1007/BF02484269

19. Mingyu, X., Wenchang, T.: Theoretical analysis of the velocity field, stress field and vortex sheet of generalized second order fluid with fractional anomalous diffusion. Sci. China Ser. A, Math. 44(11), 1387-1399 (2001). https://doi.org/10.1007/BF02877067

20. Shen, F., Tan, W., Zhao, Y., Masuoka, T.: The Rayleigh-Stokes problem for a heated generalized second grade fluid with fractional derivative model. Nonlinear Anal., Real World Appl. 7, 1072-1080 (2006). https://doi.org/10.1016/j.nonrwa.2005.09.007

21. Mahmood, A., Parveen, S., Ara, A., Khan, N.A.: Exact analytic solutions for the unsteady flow of a non-Newtonian fluid between two cylinders with fractional derivative model. Commun. Nonlinear Sci. Numer. Simul. 14(8), 3309-3319 (2009). https://doi.org/10.1016/j.cnsns.2009.01.017

22. Shen, M., Chen, S., Liu, F.: Unsteady MHD flow and heat transfer of fractional Maxwell viscoelastic nanofluid with Cattaneo heat flux and different particle shapes. Chin. J. Phys. 56(3), 1199-1211 (2018). https://doi.org/10.1016/j.c.jph.2018.04.024 
23. Zhang, Y., Zhao, H., Liu, F., Bai, Y.: Analytical and numerical solutions of the unsteady 2D flow of MHD fractional Maxwell fluid induced by variable pressure gradient. Comput. Math. Appl. 75(3), 965-980 (2018). https://doi.org/10.1016/j.camwa.2017.10.035

24. Aman, S., Al-Mdallal, Q., Khan, I.: Heat transfer and second order slip effect on MHD flow of fractional Maxwell fluid in a porous medium. J. King Saud Univ., Sci. 32, 450-458 (2018). https://doi.org/10.1016/j.jksus.2018.07.007

25. Atangana, A., Baleanu, D.: New fractional derivatives with nonlocal and non-singular kernel: theory and application to heat transfer model. arXiv preprint (2016). arXiv:1602.03408

26. Atangana, A., Baleanu, D.: Caputo-Fabrizio derivative applied to groundwater flow within confined aquifer. J. Eng. Mech. 143(5), D4016005 (2017). https://doi.org/10.1061/(ASCE)EM.1943-7889.0001091

27. Aliyu, A.I., Inc, M., Yusuf, A., Baleanu, D.: A fractional model of vertical transmission and cure of vector-borne diseases pertaining to the Atangana-Baleanu fractional derivatives. Chaos Solitons Fractals 116, 268-277 (2018). https://doi.org/10.1016/j.chaos.2018.09.043

28. Gómez-Aguilar, J.F., López-López, M.G., Alvarado-Martinez, V.M., Baleanu, D., Khan, H.: Chaos in a cancer model via fractional derivatives with exponential decay and Mittag-Leffler law. Entropy 19(12), 681 (2017). https://doi.org/10.3390/e19120681

29. Jan, S.A.A., Ali, F., Sheikh, N.A., Khan, I., Saqib, M., Gohar, M.: Engine oil based generalized Brinkman-type nano-liquid with molybdenum disulphide nanoparticles of spherical shape: Atangana-Baleanu fractional model. Numer. Methods Partial Differ. Equ. 34(5), 1472-1488 (2018). https://doi.org/10.1002/num.22200

30. Owolabi, K.M., Atangana, A.: On the formulation of Adams-Bashforth scheme with Atangana-Baleanu-Caputo fractional derivative to model chaotic problems. Chaos, Interdiscip. J. Nonlinear Sci. 29(2), 023111 (2019). https://doi.org/10.1063/1.5085490

31. Saad, K.M., Khader, M.M., Gómez-Aguilar, J.F., Baleanu, D.: Numerical solutions of the fractional Fisher's type equations with Atangana-Baleanu fractional derivative by using spectral collocation methods. Chaos, Interdiscip. J. Nonlinear Sci. 29(2), 023116 (2019). https://doi.org/10.1063/1.5086771

32. Inc, M., Yusuf, A., Aliyu, A.I., Baleanu, D.: Investigation of the logarithmic-KdV equation involving Mittag-Leffler type kernel with Atangana-Baleanu derivative. Phys. A, Stat. Mech. Appl. 506, 520-531 (2018) https://doi.org/10.1016/j.physa.2018.04.092

33. Zafar, A.A., Fetecau, C.: Flow over an infinite plate of a viscous fluid with non-integer order derivative without singular kernel. Alex. Eng. J. 55(3), 2789-2796 (2016). https://doi.org/10.1016/j.aej.2016.07.022

34. Khan, H., Khan, A., Jarad, F., Shah, A.: Existence and data dependence theorems for solutions of an ABC-fractional order impulsive system. Chaos Solitons Fractals 131, 109477 (2020)

35. Sheikh, N.A., Ali, F., Khan, I., Gohar, M.: A theoretical study on the performance of a solar collector using $\mathrm{CeO}_{2}$ and $\mathrm{Al}_{2} \mathrm{O}_{3}$ water based nanofluids with inclined plate: Atangana-Baleanu fractional model. Chaos Solitons Fractals 115 , 135-142 (2018)

36. Bas, E., Ozarslan, R.: Real world applications of fractional models by Atangana-Baleanu fractional derivative. Chaos Solitons Fractals 116, 121-125 (2018). https://doi.org/10.1016/j.chaos.2018.09.019

37. Khan, H., Jarad, F., Abdeljawad, T., Khan, A.: A singular ABC-fractional differential equation with $p$-Laplacian operator. Chaos Solitons Fractals 129, 56-61 (2019). https://doi.org/10.1016/j.chaos.2019.08.017

38. Khan, A., Khan, H., Gómez-Aguilar, J.F., Abdeljawad, T.: Existence and Hyers-Ulam stability for a nonlinear singular fractional differential equations with Mittag-Leffler kernel. Chaos Solitons Fractals 127, 422-427 (2019). https://doi.org/10.1016/j.chaos.2019.07.026

39. Khan, H., Gómez-Aguilar, J.F., Alkhazzan, A., Khan, A.: A fractional order HIV-TB coinfection model with nonsingular Mittag-Leffler law. Math. Methods Appl. Sci. 43(6), 3786-3806 (2020)

40. Khan, I.: New idea of Atangana and Baleanu fractional derivatives to human blood flow in nanofluids. Chaos, Interdiscip. J. Nonlinear Sci. 29(1), 013121 (2019). https://doi.org/10.1063/1.5078738

41. Mittag-Leffler, G.: Sopra la funzione E $\alpha(x)$. Rend. Accad. Lincei, Ser. 5(13), 3-5 (1904)

\section{Submit your manuscript to a SpringerOpen ${ }^{\circ}$ journal and benefit from:}

- Convenient online submission

- Rigorous peer review

- Open access: articles freely available online

- High visibility within the field

- Retaining the copyright to your article

Submit your next manuscript at $\boldsymbol{s p r i n g e r o p e n . c o m ~}$ 